\title{
Stundenlohnstudie: stabile Ergebnisse
}

\section{Beatrice Brunnera, Esther Kraft ${ }^{b}$}

a WIG/ZHAW, Co-Leiterin Gesundheitsökonomische Forschung, Winterthur; ${ }^{b}$ FMH, Leiterin Abteilung Daten, Demographie und Qualität (DDQ), Bern

\section{Einleitung}

Zu Beginn des Jahres hat die ZHAW im Auftrag der FMH eine Studie zu den Stundenlöhnen von Ärztinnen und Ärzten im Vergleich zu anderen akademischen Berufsgruppen veröffentlicht [1]. Nun liegt die überarbeitete Auflage der Studie vor [2]. Da für die FMH Transparenz und eine verlässliche Datengrundlage die Basis für eine sachliche, faktenbasierte und lösungsorientierte Diskussion der Ärzteeinkommen sind, präsentiert der folgende Artikel die neuen Ergebnisse und nimmt Bezug zur Erhebung MAS (Medical Ambulatory - Structure) des Bundesamtes für Statistik BFS.

\section{Ziel der Studie "Gleiches mit Gleichem vergleichen"}

Das Ziel der Stundenlohnstudie ist die Berechnung der effektiven Stundenlöhne der Ärzteschaft in der Schweiz unter Berücksichtigung der tatsächlichen Wo-

\section{Das Ziel der Stundenlohnstudie ist} die Berechnung der effektiven Stundenlöhne der Ärzteschaft in der Schweiz.

chenarbeitszeit, der Anzahl der bezogenen Ferientage und der kantonalen Feiertage (zwischen Montag und Freitag). Auf derselben Datenbasis werden auch die Stundenlöhne anderer akademischer Berufsgruppen berechnet und mit denjenigen der Ärztinnen und Ärzte verglichen.

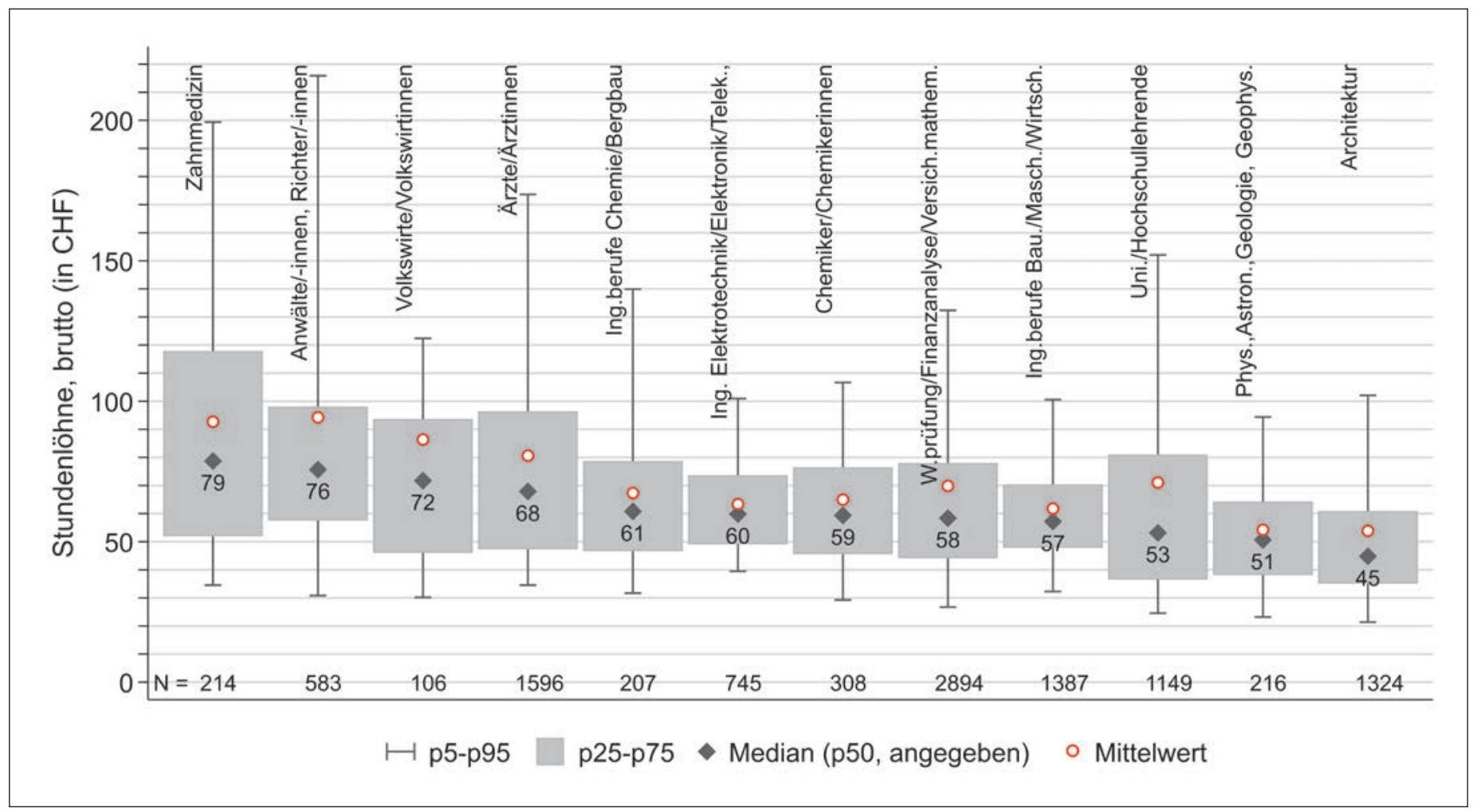

Abbildung 1: Stundenlöhne bzw. -einkommen für verschiedene akademische Berufe (2014-2017). 


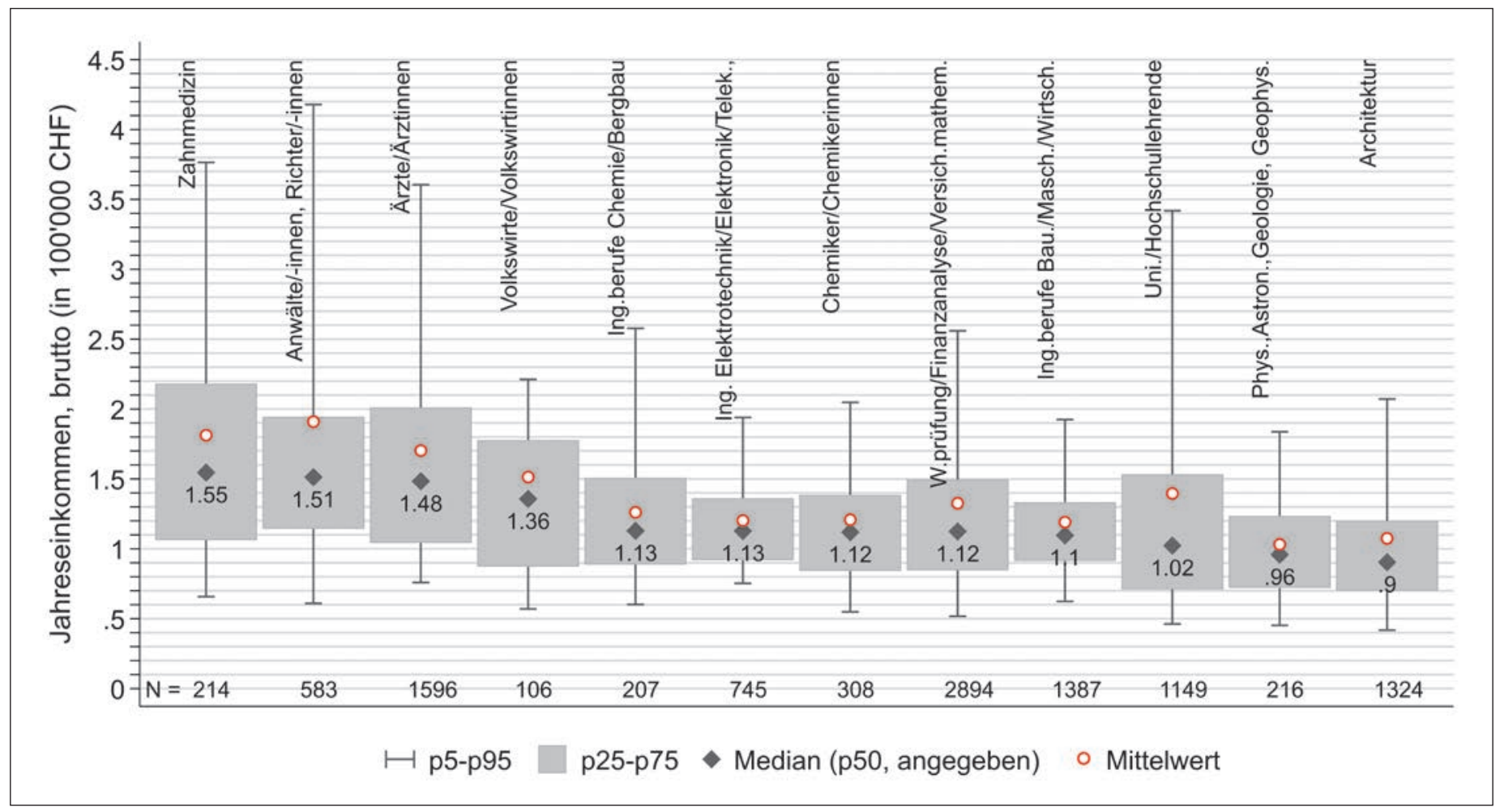

Abbildung 2: Jahreseinkommen (VZÄ std.) für verschiedene akademische Berufe (2014-2017).

Im Januar 2019 wurden die Resultate der ersten Auflage dieser Studie veröffentlicht [1]. In der zweiten Auflage [2] werden durch Nutzung zusätzlicher Daten erstens die Ärzte vollständiger identifiziert und dadurch die Repräsentativität verbessert, und zweitens die Stundenlöhne präziser abgebildet.

Die Studie verwendet die Schweizer Arbeitskräfteerhebung (SAKE) als Grundlage. Die SAKE ist eine jährliche vom BFS durchgeführte Stichprobenerhebung in Form einer Personenbefragung, die pro Jahr ca. 40000 Erwerbspersonen erfasst. Sie enthält unter anderem Informationen zu Beruf, Erwerbsstatus, Wirtschaftszweig des Arbeitsbetriebes, Einkommen, den bezogenen Ferientagen sowie den tatsächlich geleisteten

Die SAKE ist eine jährliche vom BFS durchgeführte Stichprobenerhebung in Form einer Personenbefragung.

wöchentlichen Arbeitsstunden. Wir danken dem BFS für die technischen Bemerkungen, die im Rahmen der ersten Version der Studie eingegangen sind und welche bei der zweiten Auflage berücksichtigt worden sind.

\section{Ergebnisse der Stundenlohnstudie}

Das standardisierte Brutto-Jahreseinkommen der in der SAKE erfassten Ärzte lag 2014-2017 im Median bei
148000 CHF, wie die Abbildung 2 zeigt. Sie liegen damit unter den betrachteten Berufsgruppen an dritter Stelle. Das höchste Medianeinkommen weisen die Zahnärzte mit $155000 \mathrm{CHF} / \mathrm{Jahr}$ auf, gefolgt von den Anwälten und Richtern mit 151000 CHF/Jahr. Die Unterschiede akzentuieren sich, wenn die Stundenlöhne verglichen

\section{Die Ärzte liegen mit einem Median-Stundenlohn von 78 CHF erneut auf dem vierten Platz.}

werden. Die Ärzte liegen mit einem Median-Stundenlohn von 68 CHF auf dem vierten Platz (Abb. 1).

Weil die Repräsentativitätsanalyse zeigte, dass junge Ärzte (v.a. unter 35 Jahren) in der SAKE überrepräsentiert und damit die Ärztelöhne unterschätzt sind, wurde derselbe Vergleich nochmals für Personen ab einem Alter von 35 Jahren durchgeführt. Dadurch wird ein Grossteil der Assistenzärzte ausgeschlossen, da das Durchschnittsalter bei Erwerb des Facharzttitels bei ungefähr 36 Jahren liegt. Das standardisierte Brutto-Jahreseinkommen der in der SAKE erfassten Ärzte ab 35 Jahren lag im Median bei 168000 CHF (Abb. 4).

Damit liegen sie an erster Stelle, knapp vor den Zahnärzten (166000 CHF/Jahr) und den Anwälten und Richtern (163000 CHF/Jahr) und Volkswirten (162000 CHF/ Jahr). Eine andere Reihenfolge ergibt sich für die Stundenlöhne. Die Ärzte liegen mit einem Median-Stun- 
denlohn von 78 CHF erneut auf dem vierten Platz. An erster Stelle stehen die Zahnärzte mit $88 \mathrm{CHF} / \mathrm{h}$, gefolgt von den Volkswirten mit $86 \mathrm{CHF} / \mathrm{h}$ und den Anwälten und Richtern mit $82 \mathrm{CHF} / \mathrm{h}$ (Abb. 3).

Beide Vergleiche zeigen, dass das relativ hohe Jahreseinkommen der Ärzte im Vergleich zu anderen akademischen Berufsgruppen u.a. durch überdurchschnittliche Arbeitszeiten zustande kommt. Im Median arbeiteten die Ärzte/Ärztinnen ab 35 Jahren 49,5 Stunden pro Woche, was im Vergleich zu den anderen Berufen einem deutlich höheren Arbeitsvolumen entspricht.

\section{Ergebnisse MAS 2017}

Das Bundesamt für Statistik (BFS) publizierte am 24.10.2019 die Ergebnisse aus der Erhebung MAS zu den Strukturdaten der Arztpraxen und ambulanten Zentren aus dem Jahr 2017 [3]. Die Erhebung erfolgte auf

\section{Die MAS-Erhebung weist für die in Einzel- praxen tätigen Ärzte ein Nettoeinkommen von 154000 CHF aus.}

Basis von Artikel 23 und Artikel 59a des Krankenversicherungsgesetzes (KVG) und in Anwendung des Bundesstatistikgesetzes (BStatG). Sie diente statistischen und aufsichtsrechtlichen Zwecken. Im Vergleich zur ersten Erhebung hat sich die Kontakt- und Antwortquote wesentlich verbessert (von 70 auf $82 \%$ bzw. von 52 auf 68\%). Damit erlaubte die MAS-Erhebung 2017 eine fundierte Analyse der Finanzsituation der Arztpraxen und ambulanten Zentren in der Schweiz. Die Analyse weist für die in Einzelpraxen tätigen Ärzte einen Gesamtertrag (Median) von 428000 CHF und einen Ge-

Vom Ertrag aus Praxistätigkeit stammten im Schnitt $85 \%$ aus der obligatorischen Krankenversicherung.

samtaufwand von 263000 CHF (Median) aus. Ihr Nettoeinkommen betrug somit 154000 CHF (Median) im Jahr 2017. Vom Ertrag aus Praxistätigkeit stammten im Schnitt 85\% aus der obligatorischen Krankenversicherung [3]. Die MAS-2017-Ergebnisse weisen für die Ärztinnen und Ärzte eine Wochenarbeitszeit von durchschnittlich 40 Stunden aus, was einem 75\%-Pensum entspricht. Dies bedeutet, dass die Ärztinnen und Ärzte eine rund ein Viertel höhere Wochenarbeitszeit ausweisen, als die übliche Wochenarbeitszeit in der Schweiz beträgt. Dies deckt sich mit den Ergebnissen der Stundenlohnstudie, welche zeigt, dass die Ärzte knapp 18\% mehr arbeiten als die anderen akademischen Berufsgruppen. Gemäss den in der Stundenlohnstudie ausgewiesenen Ferien der Ärztinnen und Ärzte kann bei den MAS-2017-Daten approximativ

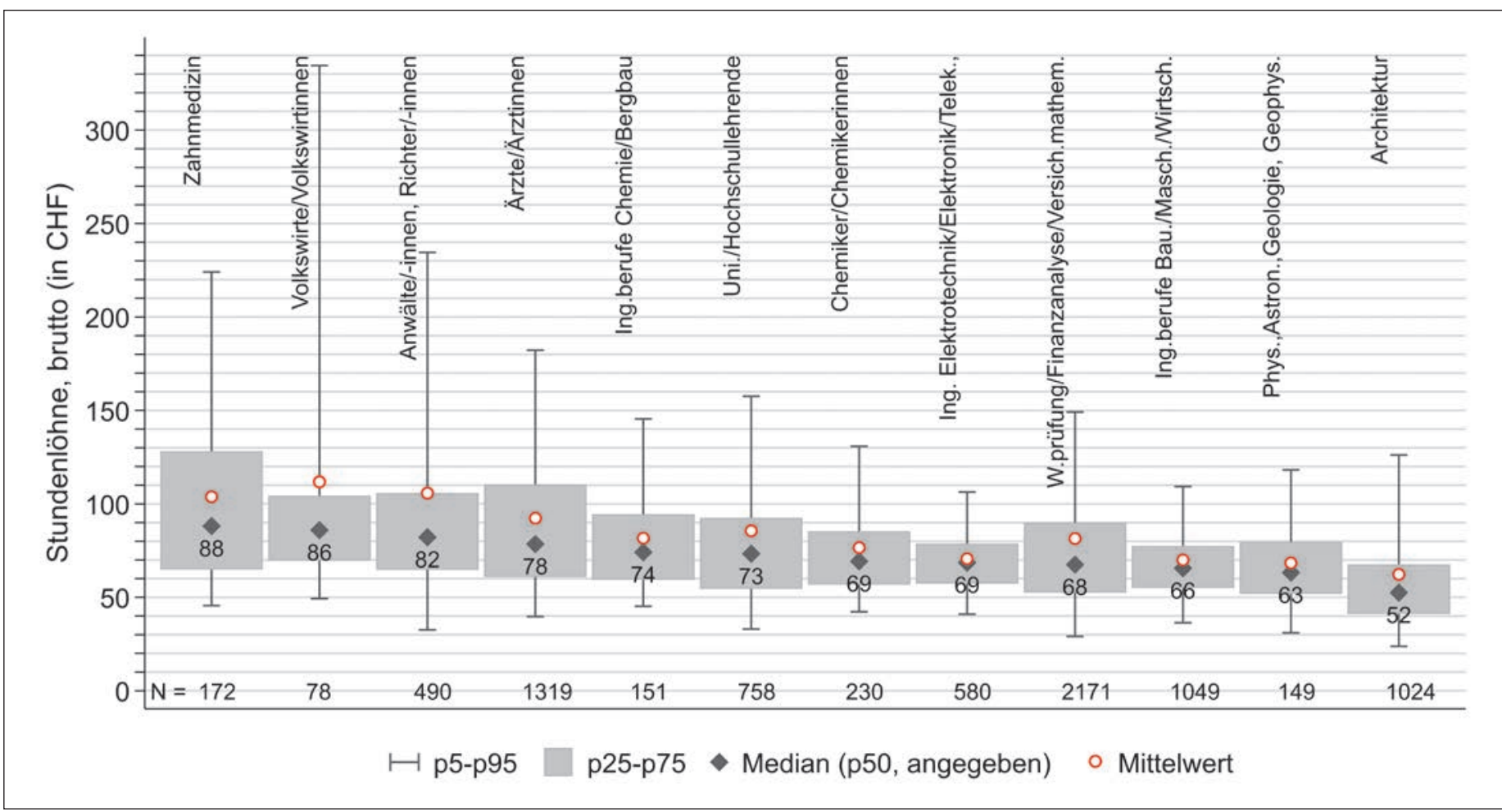

Abbildung 3: Stundenlöhne bzw. -einkommen für verschiedene akademische Berufe (2014-2017), ab 35 Jahren. 


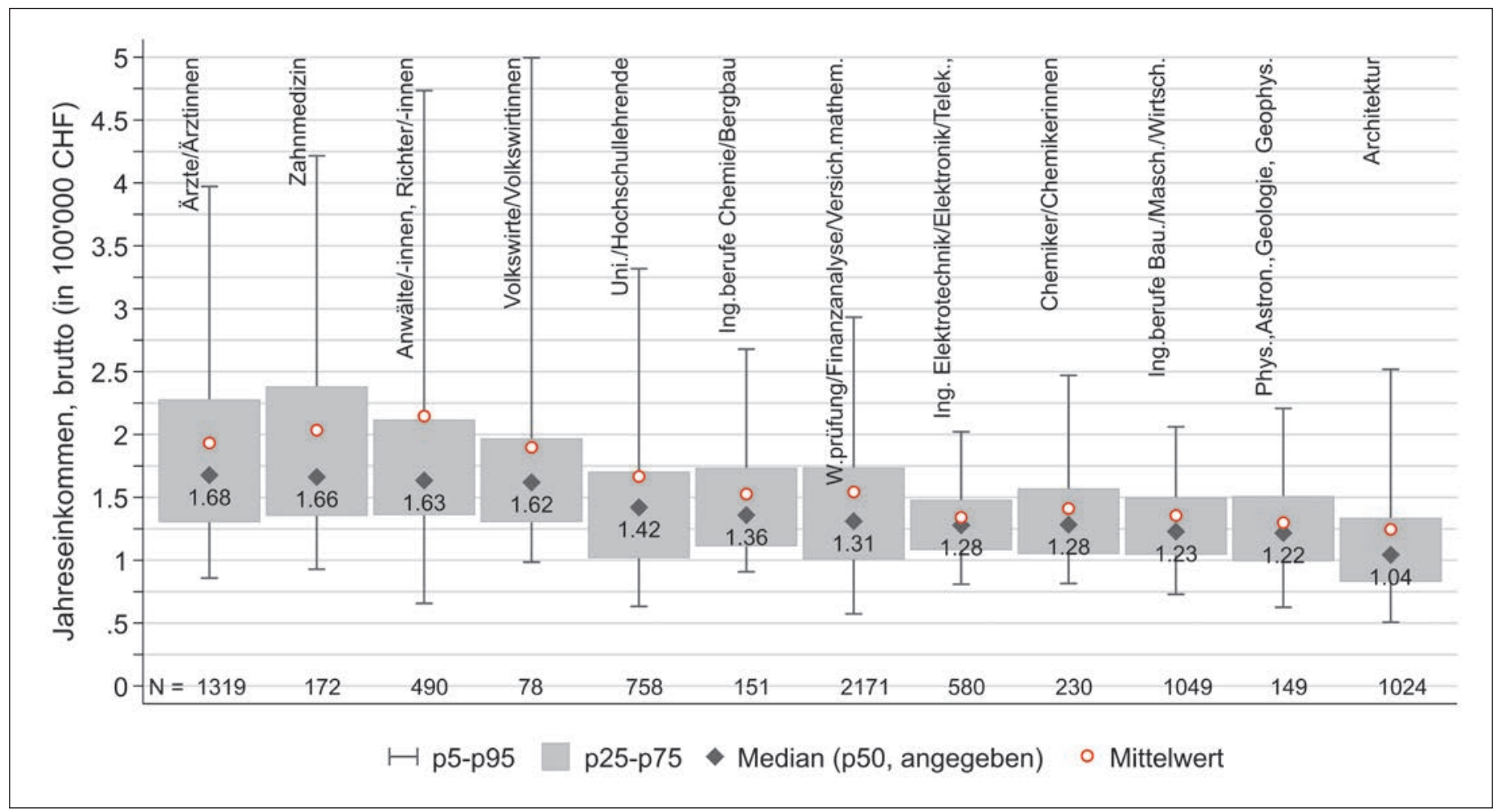

Abbildung 4: Jahreseinkommen (VZÄ std.) für verschiedene akademische Berufe (2014-2017), ab 35 Jahren.

Beatrice Brunner

WIG/ZHAW

Gertrudstrasse 15

CH-8401 Winterthur

beatrice.brunner[at]zhaw.ch

Esther Kraft

FMH

Elfenstrasse 18

CH-3000 Bern 15

esther.kraft[at]fmh.ch von einem Netto-Stundenlohn in Einzelpraxen von ca. $84 \mathrm{CHF}$ ausgegangen werden.

\section{Fazit und Ausblick}

Die überarbeitete Stundenlohnstudie berechnet und vergleicht die Stundenlöhne von Ärzten und anderen Berufsgruppen in der Schweiz. Damit wird innerhalb eines statistisch klar definierten Kollektivs (SAKE) «Gleiches mit Gleichem» verglichen.

Die MAS-2017-Erhebung des BFS, welche die Wochenarbeitszeit neu auch erfasst, ermöglicht erstmals die Validierung der Resultate der Stundenlohnstudie. Wie der approximative Vergleich zeigt, sind die Ergebnisse vergleichbar und ermöglichen somit eine sachliche, faktenbasierte und lösungsorientierte Diskussion.

Literatur

1 Brunner B, Kraft E. Stundenlohnstudie - Vergleich zwischen Ärzten und anderen Berufsgruppen. Schweiz Ärzteztg. 2019;100(6):158-9.

2 Studie Brunner: https://digitalcollection.zhaw.ch/bitstream/11475/18477/1/2019 Stundenlohn\%20Aerzte Brunner.pdf 3 MAS 2017-Ergebnisse, Website BFS; https://www.bfs.admin.ch/ bfs/de/home/statistiken/gesundheit/gesundheitswesen/arztpraxen.html

Bildnachweise

WIG/ZHAW 